\title{
Théâtres de trois continents - en français : une autre genèse?
}

Présentation

\section{Florence Davaille}

\section{(2) OpenEdition} Journals

Édition électronique

URL : http://journals.openedition.org/coma/749

DOI : $10.4000 /$ coma.749

ISSN : 2275-1742

Éditeur

Institut des textes \& manuscrits modernes (ITEM)

\section{Référence électronique}

Florence Davaille, "Théâtres de trois continents - en français : une autre genèse ? », Continents manuscrits [En ligne], 7 | 2016, mis en ligne le 15 décembre 2016, consulté le 23 septembre 2020. URL : http://journals.openedition.org/coma/749 ; DOI : https://doi.org/10.4000/coma.749

Ce document a été généré automatiquement le 23 septembre 2020.

\section{(c) (i) $\odot$}

Continents manuscrits - Génétique des textes littéraires - Afrique, Caraîbe, dispora est mis à disposition selon les termes de la licence Creative Commons Attribution - Pas d'Utilisation Commerciale - Pas de Modification 4.0 International. 


\section{Théâtres de trois continents - en français : une autre genèse ?}

Présentation

Florence Davaille

1 Ce dossier présente une réflexion sur les genèses du texte théâtral à partir de cinq cas : le Martiniquais Aimé Césaire et la Tragédie du Roi Christophe, le Franco-américain (du Sud) Jules Supervielle et Bolivar, le Franco-tunisien Albert Memmi et Le Prince (texte inédit, que l'auteur est d'ailleurs en train de retravailler), le Canadien du Manitoba Marc Prescott et Sex, lies, et les Franco-Manitobains, et le Canadien d'origine irakienne Naïm Kattan, qui a publié en 2015 un volume de Théâtre. ${ }^{1}$

2 On avait coutume de nommer ce théâtre «francophone », pour le distinguer d'un théâtre de France qui s'écrit lui-aussi en français. On sait quelle polémique entoure cette façon d'utiliser l'adjectif «francophone ", quand il n'est pas pris dans sa simple acception linguistique: il s'agirait de marquer ainsi une éternelle "différence » (parfois perçue comme une différence de niveau) et d'envisager ces littératures comme « jeunes » et n'ayant pas atteint un degré de maturité suffisant. Le titre de ce dossier a donc choisi de parler d'un théâtre "en français", pour éviter le mot piégé de «francophone» (quand il ne se cantonne pas à sa simple acception linguistique). Le dossier adopte un double parti pris. Pour dire les choses autrement, on a fait le choix de parler de "théâtres de trois continents », ces derniers renvoyant ici à l'Europe, aux Amériques (du Nord, du Sud, et Caraïbes) et à l'Afrique (celle du Maghreb, en l'occurrence). Le titre du dossier se met ainsi en accord avec celui de la revue. On pourrait donc ajouter ultérieurement des exemples concernant le continent asiatique, ou cette "autre Afrique» - subsaharienne cette fois -, ou les "Îles» (Océan indien, archipel polynésien). L'expression a l'avantage, néanmoins, de rompre avec cette "conception pyramidale» de l'espace francophone (la France au sommet, et plus particulièrement Paris, et "les autres » plus loin, et en-dessous). Elle permet de s'intéresser à tous ceux qui parlent français, y compris ... les « Français de France ». Elle permet de souligner que le français existe ailleurs qu'en France et qu'il est pratiqué dans d'autres conjonctures culturelles qui en changent la réalité linguistique et le vécu. 
Ainsi, ce dossier fait aussi le choix concerté d'inclure un cas (limite) symptomatique : Jules Supervielle, que l'on peut à la fois considérer comme "français » et/ou « américain francophone » - ce qui n'aurait pas déplu à cet écrivain qui se disait « grand constructeur de ponts dans l'espace ». Inclure Supervielle dans le dossier pourrait donc bien être un deuxième parti pris qui montre que, dans la période contemporaine, cette distinction est de plus en plus caduque.

3 On présente ici des "francophonies " incluant donc la France (à travers le cas de Supervielle), sans distinguer non plus «francophonie du Nord» et «francophonie du Sud», pour dépasser une certaine vision qui séparait implicitement, par cette dichotomie, les pays riches des pays pauvres, les colons des colonisés. La distinction a pu être utile, car elle disait tout haut une domination qu'il fallait définitivement dénoncer et dépasser. Le présent dossier, qui s'appuie sur quelques cas de théâtre contemporain, propose plutôt une vision de la «francophonie » qui sort de l'approche "post-coloniale", selon ce qui se passe dans la période contemporaine depuis les années 1990, et qui a été expliqué par de nombreux auteurs dits « francophones », tels Dany Laferrière (qui voyage en français, même au Japon), ou Maryse Condé (qui ne parle pas le créole, mais le Maryse Condé), ou Anna Moï (qui n'écrit pas seulement des romans sur le Viêt-Nam), etc... L'intérêt du présent corpus est justement qu'il parcourt une période qui va des années précédant la Deuxième Guerre mondiale à aujourd'hui et permet, d'une certaine manière, de manifester cette évolution. On s'intéresse donc ici à une littérature "des continents en français » (incluant la littérature française), où le rapport $\mathrm{du}$ texte au contexte socio-politique ne se joue plus dans l'espace d'une confrontation entre la France et ses "ailleurs francophones». On émettra donc l'hypothèse d'une possible différence entre la genèse de la Tragédie du Roi Christophe de Césaire, qui se jouerait précisément toujours dans l'espace d'une confrontation avec la France, et Sex, lies, et les Franco-Manitobains de Prescott, une pièce contemporaine qui ne peut pas se lire dans cette confrontation, mais dans celle du français et de l'anglais au Canada.

4 La genèse de ce type de théâtre change-t-elle par rapport à la genèse d'un théâtre de France en français? Plusieurs réponses sont évidemment possibles.

5 Première réponse à la question : NON, dans tous ces cas, on retrouvera la complexité de la genèse théâtrale, ainsi que Françoise Simonet-Tenant en rappelle les paramètres dans l'article liminaire du dossier. Diversité des documents, atténuation de l'instance auctoriale, interprétation des différents metteurs en scène, malléabilité d'une œuvre existant dans l'espace éphémère de la représentation : quelles que soient les cultures ici représentées, le travail du théâtre est celui d'une mise en espace d'un propos, ce qui change les paramètres de la genèse, en comparaison avec celle des autres genres littéraires. Les cas présentés ici font l'objet en tout cas d'une analyse esthétique, comme tout texte de théâtre. Le corpus «francophone » n'est plus lu uniquement selon des perspectives socio-historiques. Et donc, si l'on cherchait une différence dans la façon de concevoir une pièce et sa représentation, il faudrait sans doute s'orienter vers une autre direction: le cas où la pièce se jouerait dans une culture où la tradition orale domine. Le corpus retenu ici n'en présente pas, et la comparaison avec la genèse d'un théâtre conçu en contexte de tradition orale est encore à faire. À cet égard, on se souviendra, par exemple, de l'intérêt que le théâtre balinais avait suscité chez Artaud. On pourrait aussi s'orienter, en comparaison, vers l'étude de la genèse du théâtre d'improvisation tel qu'il est pratiqué aujourd'hui. 
6 Deuxième réponse à la question : OUI, car le français est utilisé au cœur d'autres enjeux culturels. La question du bilinguisme en Alberta en est un exemple patent. Les réécritures sont ici directement motivées par un contexte linguistique spécifique.

7 Troisième réponse à la question : OUI, car l'arrière-plan socio-historique change - mais la relation elle-même du texte à ce contexte socio-historique se joue de la même façon, on peut le supposer, en France ou dans d'autres pays qui parlent français, ou même d'autres langues (une "socio-philosophie» pourrait nous éclairer sur ce point). Le texte théâtral ici est de fait en rapport, selon les cas, avec le discours sur le colonialisme (Césaire), le contexte de la guerre d'Espagne imminente et la représentation d'un des « libérateurs » de l'Amérique (Supervielle), la question de la femme et de sa possible action politique (?) point d'interrogation à supprimer dans le contexte du Maghreb (Memmi), la tradition féministe nord-américaine utilisant les motifs de la sorcière et du sabbat (Brassard mettant en scène Albertine en Cinq Temps de Tremblay), À SUPPRIMER la difficile acceptation du bilinguisme vraiment pratiqué au Manitoba, dans un Canada qui en fait pourtant la publicité officielle (Prescott), la question actuelle de la migration des populations, dans une pièce comme Le Seuil de la promesse (Kattan).

8 Dans la mesure où le contexte socio-historique dans lequel chacune de ces pièces est écrite est important, les articles sont présentés de façon à respecter la chronologie approximative des genèses de ces différentes pièces.

9 Daniel Delas revient ici sur les abondantes réécritures que présente le théâtre de Césaire et explore cette hypothèse : c'est finalement la question du leader noir des indépendances afro-antillaises et plus généralement celle de la libération de l'homme, qui hante Césaire pendant plus de trente ans et fait graviter l'ensemble de son œuvre, non seulement théâtrale, mais poétique et argumentative. Ce faisant, Césaire cherche à créer « un théâtre qui agit philosophiquement au niveau de l'homme tout entier ».

10 La pièce Bolivar de Supervielle, jouée en 1936 à la Comédie-Française et reprenant l'histoire de l'un des plus fameux «libérateurs » des Amériques, permet de montrer comment le récit (roman ou nouvelle) est chez certains auteurs la source du texte théâtral, tandis que celui-ci se rend indispensable parce qu'il suscite le rêve d'une expression "totale» qui achèverait le mouvement créateur. Dans le cas de Bolivar, l'analyse génétique doit donc envisager un parcours passant du récit, au théâtre, puis à l'opéra, puisque la pièce fut ainsi montée en 1950 sur une musique de Darius Milhaud.

11 Hervé Sanson explore, quant à lui, la genèse de la seule pièce de théâtre d'Albert Memmi connue à ce jour et pour l'instant inédite. Son analyse s'appuie en particulier sur la «documentation provisionnelle » ayant nourri la pièce, ce que Memmi nomme son "garde-manger». La pièce, travaillée à la fin des années 1960 et au début des années 1970, permet de comprendre comment espace scénique et texte sont intrinsèquement liés dans certaines écritures dramaturgiques. Elle aborde sous cette autre forme les thèmes chers à Memmi : l'exercice du pouvoir et la place des femmes dans les sociétés arabes.

12 Louise Ladouceur analyse le cas d'une pièce de théâtre de Marc Prescott, un dramaturge bilingue de l'Ouest canadien, maîtrisant à la fois le français et l'anglais. La pièce étudiée met en scène une parole mêlant ces deux langues, ainsi qu'on peut l'entendre dans le Manitoba contemporain (1993-2013). C'est toute la relation entre les deux langues et les deux communautés dans cette région de l'Ouest du Canada qui est donc mise en question ici. L'étude des différentes révisions du texte entre les 
représentations de 1993, à Saint-Boniface au Manitoba, et de 2013, au Québec, permet de comprendre tous les enjeux politiques, sociaux et culturels que recouvre la question d'un «bilinguisme » affiché mais plus ou moins bien assumé. Les différentes révisions du texte en fonction des publics concernés (le Manitoba, le Québec) montrent aussi comment dans différentes régions francophones du Canada se joue le rapport aux deux langues officielles du pays.

13 À la fin du dossier, on trouvera un témoignage de Naïm Kattan - écrivain originaire de Bagdad, vivant depuis 1954 entre le Québec et Paris - sur son rapport au théâtre. L'auteur, plus connu pour ses romans et son œuvre de critique, explique ici selon quelles nécessités émerge l'écriture théâtrale pour lui. Il a publié en 2015 le volume Théâtre (1970-2014) dans la collection "Franco-Amériques» des PURH (Presses de l'université de Rouen et du Havre). Cet ouvrage regroupe une vingtaine de pièces, qui furent composées dans le cadre du théâtre radiophonique de Radio-Canada dans les années 1970 ou qui sont des pièces récentes. ${ }^{2}$

\section{NOTES}

1. Ce dossier est issu d'une journée d'étude qui s'est tenue à l'université de Rouen sur les "Genèses du texte théâtral en français », et qui a fait l'objet d'une collaboration entre l'ITEM et le CEREdI (Centre Editer / Interpréter) de l'université de Rouen.

2. Lors de la journée d'étude de Rouen, Rachel Killick, professeur à l'université de Leeds, avait présenté également une communication sur la genèse d'Albertine en cinq temps du dramaturge québécois Michel Tremblay. Elle a retracé l'évolution de l'une des pièces les plus connues de Michel Tremblay et du répertoire théâtral québécois. Les différents manuscrits de la pièce, les manuscrits retravaillés par le metteur en scène André Brassard pour les représentations de 1984, son journal de bord ainsi que celui de Martine Beaulne, qui monta la pièce en 1995 à l'espace Go, et les affiches des deux spectacles ont rendu compte de la diversité des documents de genèse présents dans le cas du théâtre. Ainsi ont émergé sous un jour nouveau les enjeux d'une pièce qui transforma un simple dialogue entre deux sœurs en une confrontation surréelle entre cinq états de la même personne. Un ouvrage sur la question devrait paraître bientôt.

\section{AUTEUR}

\section{FLORENCE DAVAILLE}

Université de Rouen / ITEM 